\title{
RhoA protects the mouse heart against ischemia/reperfusion injury
}

\author{
Sunny Yang Xiang,, ${ }^{1}$ Davy Vanhoutte, ${ }^{2}$ Dominic P. Del Re,1,3 Nicole H. Purcell, ${ }^{1}$ Haiyun Ling, ${ }^{1}$ \\ Indroneal Banerjee, ${ }^{4}$ Julie Bossuyt, ${ }^{5}$ Richard A. Lang, ${ }^{6}$ Yi Zheng, ${ }^{7}$ Scot J. Matkovich, ${ }^{8}$ \\ Shigeki Miyamoto, ${ }^{1}$ Jeffery D. Molkentin, ${ }^{2}$ Gerald W. Dorn II, ${ }^{8}$ and Joan Heller Brown'
}

\begin{abstract}
${ }^{1}$ Department of Pharmacology, UCSD, San Diego, California, USA. ${ }^{2}$ Molecular Cardiovascular Biology, Cincinnati Children's Hospital Medical Center, Cincinnati, Ohio, USA. ${ }^{3}$ Cardiovascular Research Institute, University of Medicine and Dentistry of New Jersey, New Jersey Medical School, Newark, New Jersey, USA. ${ }^{4}$ Department of Medicine, UCSD, San Diego, California, USA. ${ }^{5}$ Department of Pharmacology, UCD, Davis, California, USA. ${ }^{6}$ Visual Systems Group, and 7Experimental Hematology and Cancer Biology, Cincinnati Children's Hospital Medical Center, Cincinnati, Ohio, USA. ${ }^{8}$ Department of Medicine, Washington University in St. Louis, St. Louis, Missouri, USA.
\end{abstract}

\begin{abstract}
The small GTPase RhoA serves as a nodal point for signaling through hormones and mechanical stretch. However, the role of RhoA signaling in cardiac pathophysiology is poorly understood. To address this issue, we generated mice with cardiomyocyte-specific conditional expression of low levels of activated RhoA (CA-RhoA mice) and demonstrated that they exhibited no overt cardiomyopathy. When challenged by in vivo or ex vivo ischemia/reperfusion (I/R), however, the CA-RhoA mice exhibited strikingly increased tolerance to injury, which was manifest as reduced myocardial lactate dehydrogenase (LDH) release and infarct size and improved contractile function. PKD was robustly activated in CA-RhoA hearts. The cardioprotection afforded by RhoA was reversed by PKD inhibition. The hypothesis that activated RhoA and PKD serve protective physiological functions during $I / R$ was supported by several lines of evidence. In WT mice, both RhoA and PKD were rapidly activated during I/R, and blocking PKD augmented I/R injury. In addition, cardiac-specific RhoA-knockout mice showed reduced PKD activation after I/R and strikingly decreased tolerance to I/R injury, as shown by increased infarct size and LDH release. Collectively, our findings provide strong support for the concept that RhoA signaling in adult cardiomyocytes promotes survival. They also reveal unexpected roles for PKD as a downstream mediator of RhoA and in cardioprotection against I/R.
\end{abstract}

\section{Introduction}

RhoA is the best-characterized Rho family small GTPase, acting as an intracellular signal transducer that modulates a range of cellular processes including adhesion, migration, invasion, cell cycle progression, and gene expression (1-4). In light of the multiple upstream regulators and downstream mediators of RhoA signaling, it is perhaps not surprising that its role in cardiac physiology and pathophysiology remains elusive (5). On the one hand, there are published data demonstrating that high levels of RhoA expression in cardiomyocytes can induce apoptosis (6) and cardiomyopathy $(7,8)$ and that the well-characterized RhoA effector Rho kinase (ROCK) has deleterious effects on the heart (9-12). On the other hand, expression of RhoA at a more physiological level can protect cardiomyocytes against apoptosis induced by $\mathrm{H}_{2} \mathrm{O}_{2}$ or glucose deprivation (13) and inhibiting Rho leads to caspase-3 activation and apoptosis (14). RhoA has also been implicated in adenosine-induced cardioprotection against ischemia (15). To control for the myriad contextual events that could influence the functional response to RhoA, e.g., the cellular site and developmental stage of RhoA expression and the mechanism and extent of RhoA activation, we utilized the attenuated $\alpha$ myosin heavy chain-driven ( $\alpha$ MHC-driven) tetracycline-responsive (tet-off) system to generate transgenic mice with conditional in vivo postnatal expression of modest levels of constitutively activated RhoA in cardiomyocytes. We demonstrate here that when RhoA expression is induced in adult cardiomyocytes, neither hypertrophy nor cardiomyopathy ensues. Instead, the increase in activated RhoA in cardiomyocytes confers remarkable protection

Conflict of interest: The authors have declared that no conflict of interest exists. Citation for this article: J Clin Invest. 2011;121(8):3269-3276. doi:10.1172/JCI44371. against ischemia/reperfusion (I/R) injury in vivo and in the isolated perfused heart. Conversely, cardiac-specific RhoA-knockout mice are substantially more susceptible to I/R injury. We provide further evidence suggesting that $\mathrm{PKD}$, an enzyme not heretofore implicated in cardiomyocyte survival, is a mediator of RhoA signaling and cardioprotection against I/R injury.

\section{Results}

Conditional cardiac-specific RhoA transgenic mice show no overt pathological phenotype. Conditional cardiomyocyte-specific expression of constitutively activated (Q63L) RhoA (CA-RhoA) was achieved by crossing transgenic mice expressing the RhoA transgene under the control of an attenuated tetracycline-responsive $\alpha \mathrm{MHC}$ promoter, with a mouse line expressing the tetracycline transactivator (tTA). Double-transgenic mice should express the regulated transgene in cardiomyocytes shortly after birth in the absence of tetracycline but not if animals are maintained on tetracycline (16). Doxycycline (Dox), a stable analog of tetracycline, was administered to the breeding dams and pups throughout embryonic development and until weaning ( 3 weeks of age). Two CA-RhoA lines (line 7 and line 25), which showed respectively, 2- and 5-fold increases in active RhoA in the LV at 8 weeks after Dox removal (off Dox) (Figure 1A) were extensively characterized. Cardiac structure, dimension, and functional performance were normal in both lines examined at 8 weeks and followed for 8 months off Dox (Figure 1B and Supplemental Table 1; supplemental material available online with this article; doi:10.1172/JCI44371DS1). Histological analyses revealed no overt pathology, hypertrophy, or fibrosis in hearts from either line, as assessed by H\&E and Masson's trichrome (Tri) staining (Figure 1C). Thus, expression of 
A

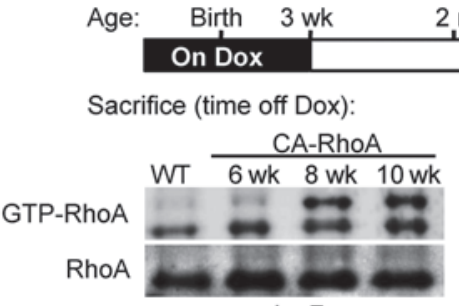

$\operatorname{Ln} 7$

$\frac{2 \text { mo }}{\text { Off Dox }}$

6 wk 8 wk $10 \mathrm{wk}$

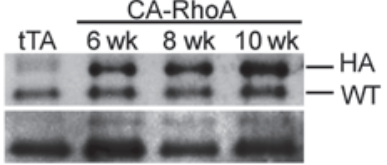

$\operatorname{Ln} 25$
B
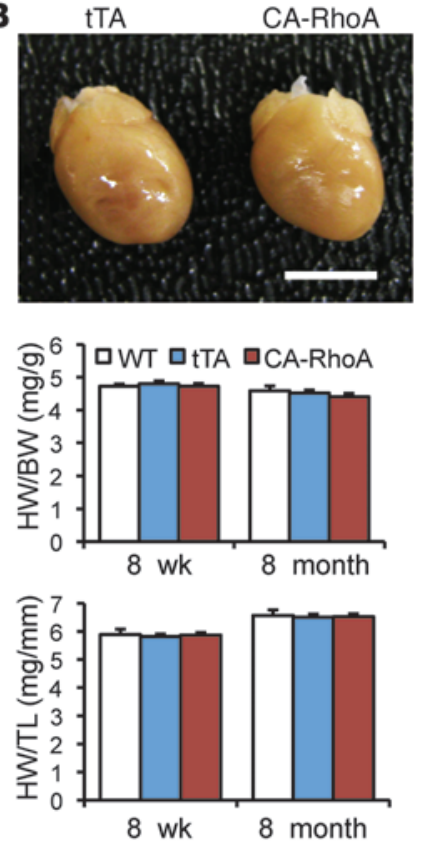

C tTA
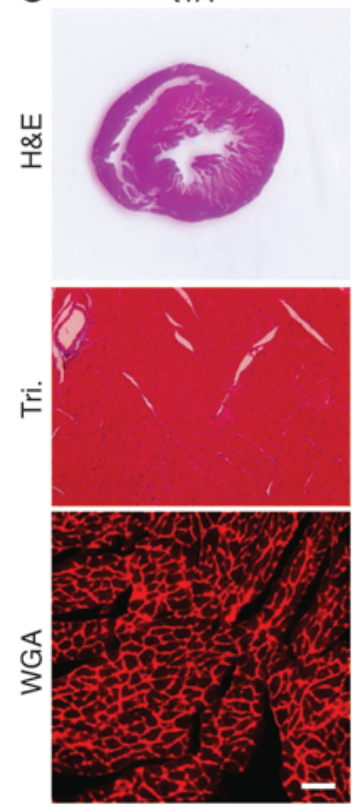

CA-RhoA
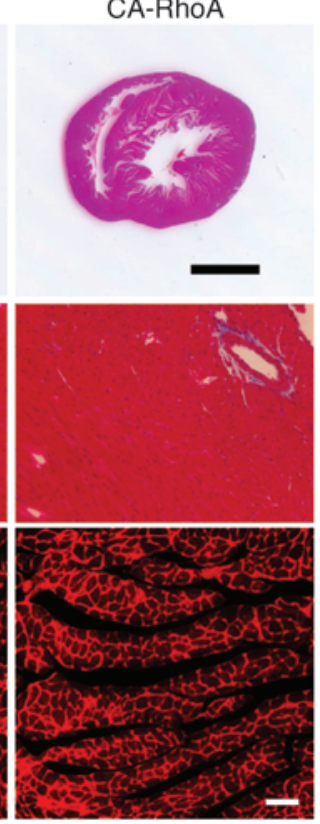

\section{Figure 1}

Characterization of conditional cardiac-specific RhoA transgenic mice. (A) Top panel: time line of Dox administration and sacrifice of animals for analysis. Bottom panel: activation and expression of endogenous (WT) and transgenic (HA-tagged) RhoA in the LV of CA-RhoA mouse hearts from lines 7 and 25 at various times after Dox removal (off Dox). (B) Representative image of hearts from tTA and CA-RhoA (line 25) mice (top panel). Scale bar: $5 \mathrm{~mm}$. Heart weight (HW) to body weight (BW) ratio and heart weight to tibial length $(T L)$ ratio from WT, tTA, and CA-RhoA mice at 8 weeks or 8 months after Dox removal. Data are shown as mean \pm SEM. (C) Representative staining of histological sections in TTA and CA-RhoA (line 25) hearts by H\&E staining (top). Scale bars: $2 \mathrm{~mm}$. Tri staining (middle) and TRITCconjugated, WGA staining of cell membranes (bottom). Scale bars: $50 \mu \mathrm{m}$. modest levels of RhoA in the adult mouse heart is not sufficient to induce hypertrophy nor is it inherently pathological.

Notably, in some initial control experiments, breeding dams and pups were not administered Dox, enabling RhoA expression during the early postnatal period. Three CA-RhoA lines (lines 7, 10, and 25), which showed 3- to 7-fold increases in active RhoA, all developed hypertrophy as assessed by heart weight to tibial length ratio (Supplemental Figure 1). Comparison with the conditional expression paradigm used in Figure 1 suggests that induction of hypertrophy in response to activated RhoA reflects signaling events that occur during heart development but not in adulthood.

$C A-R h o A$ mice show increased tolerance against $I / R$ injury in vivo and in the isolated perfused heart. Our previous work demonstrated that RhoA expression in cardiomyocytes can confer protection against oxidative stress (13). To determine whether this occurs in vivo, we examined the response to I/R injury in CA-RhoA mice. Control (WT, tTA) and CA-RhoA (line 25) mice (8 weeks off Dox) were subject to experimental myocardial I/R through ligation of the left anterior descending artery (LAD) for 1 hour, followed by removal of the ligation and reperfusion for 24 hour. The area at risk (AAR) was not different in CA-RhoA versus control littermates (Figure 2A). Strikingly, however, infarct size was $70 \%$ smaller in the CA-RhoA compared with the control hearts $(10 \% \pm 1 \%$ vs. $37 \% \pm 3 \%$ of AAR) (Figure $2 B$ ), demonstrating in vivo protection against I/R injury.

To exclude a role for endogenous systemic neurohormonal mechanisms in the cardioprotection seen in CA-RhoA mice, further studies were performed on isolated perfused hearts. Following a 30-minute period of global ischemia, LV-developed pressure (LVDP) recovered over 45 minutes of reperfusion to $64 \%$ of preischemic values in the CA-RhoA compared with only $25 \%$ in the controls. Postischemic maximum rate of contraction $(+\mathrm{dP} / \mathrm{dt})$ and maximum rate of relaxation $(-\mathrm{dP} / \mathrm{dt})$ also showed more rapid and complete recovery in CA-RhoA hearts than in control hearts (Figure 3A). To directly assess myocardial cell damage, we examined lactate dehydrogenase (LDH) accumulation in the coronary effluent during a 45-minute
A

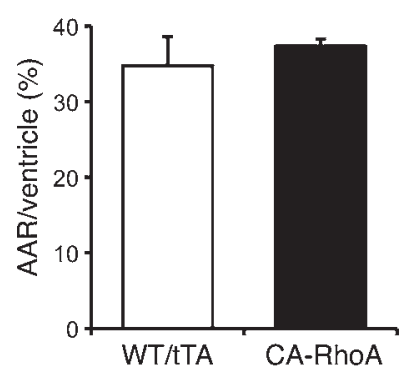

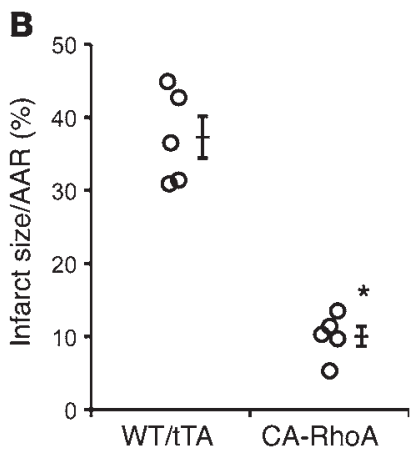

Figure 2

RhoA activation protects against $\mathrm{l} / \mathrm{R}$ injury in vivo. WT, tTA, and CA-RhoA mice (8 weeks off Dox) were subjected to in vivo I/R. (A) Quantitative analyses of AAR as a percentage of ventricular area and (B) infarct size as a percentage of AAR of hearts subjected to in vivo I/R. ${ }^{*} P<0.001$ versus WT/tTA $(n=5)$. Data are shown as mean \pm SEM. 
A
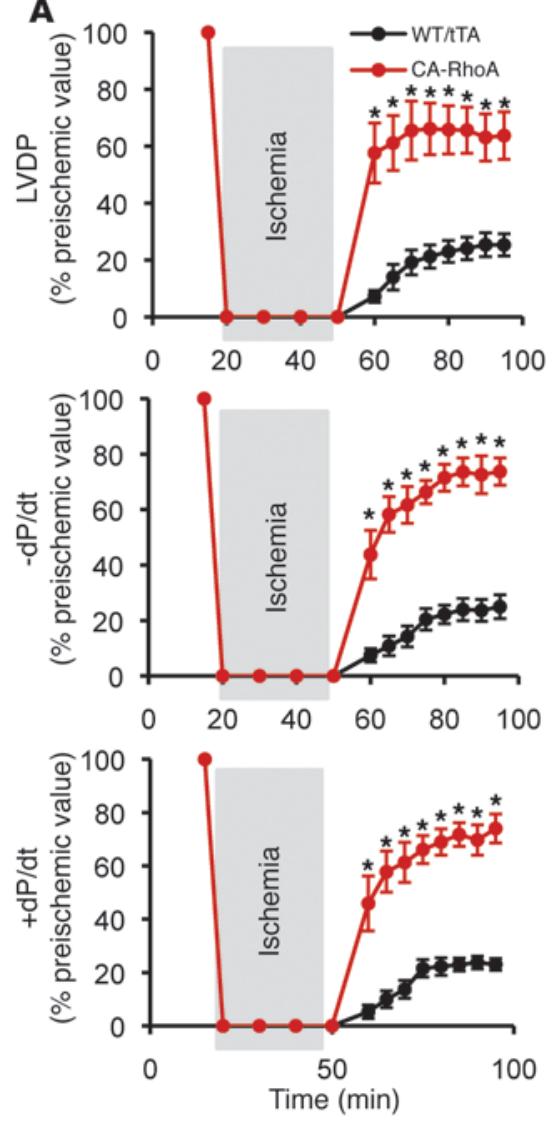

B

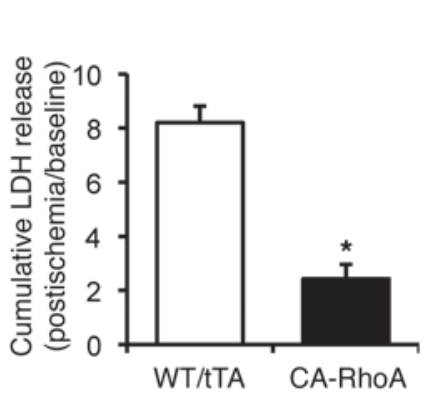

C

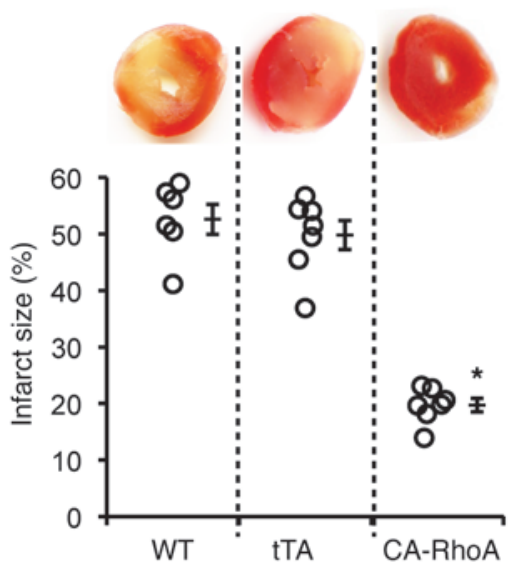

D

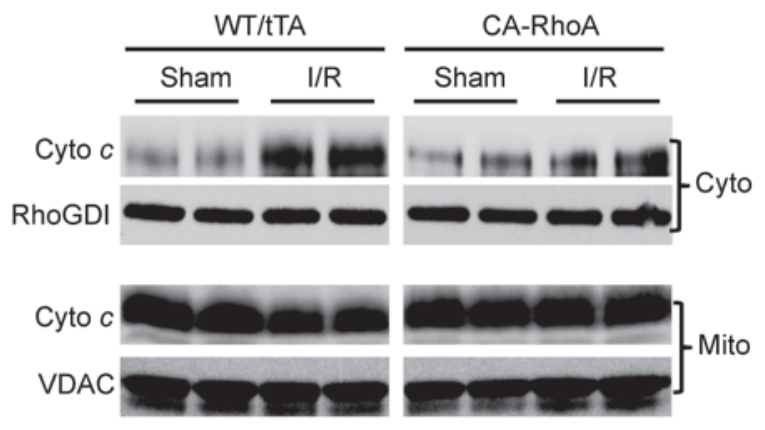

$\mathbf{E}$

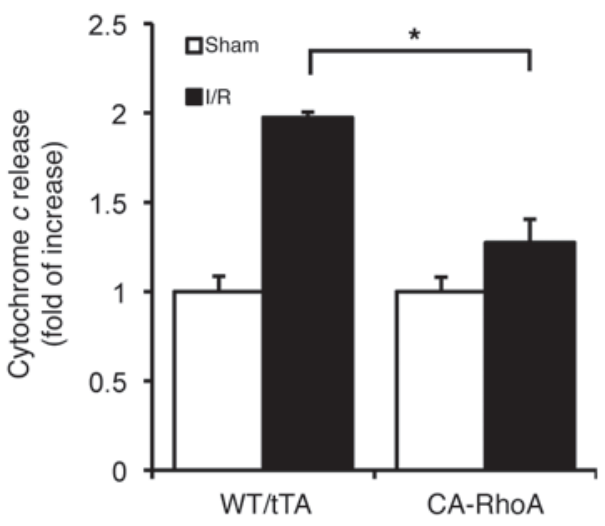

Figure 3

RhoA activation protects against I/R injury in the isolated perfused heart. WT, tTA, and CA-RhoA mice (8 weeks off Dox) were subjected to global I/R. (A) Time course recovery of contractility following ischemia measured by LVDP, $+\mathrm{dP} / \mathrm{dt}$, and $-\mathrm{dP} / \mathrm{d}$. ${ }^{*} P<0.001$ versus WT/tTA $(n=7)$. (B) LDH release to the coronary effluent following 45 minutes reperfusion. WT and tTA were not significantly different, and data were pooled together as WT/tTA. ${ }^{*} P<0.001$ versus WT/tTA $(n=6)$. (C) Representative TTC-stained heart sections (top) and quantitative analysis of infarct size (bottom). ${ }^{*} P<0.001$ versus WT or tTA $(n=6-7)$. (D and E) The LV of the WT/tTA and CA-RhoA hearts were fractioned following 30 minutes I/30 minutes R, and cytosolic (cyto) and mitochondrial (mito) cytochrome $c$ (cyto $c$ ) were analyzed by Western blots. (D) Representative blots showing cyto (top) and mito (bottom) cytochrome $c$ level. (RhoGDI and VDAC were used as cytosolic and mitochondrial loading control, respectively. VDAC was not detected in the cytosol fraction and RhoGDI was not detected in the mitochondrial fraction.) (E) Quantification of cytochrome $c$ release in the cytosolic fraction. ${ }^{*} P<0.05(n=4)$. Data are shown as mean \pm SEM.

reperfusion period. LDH release was $70 \%$ lower in CA-RhoA hearts, demonstrating a cytoprotective effect of RhoA (Figure 3B). Myocardial infarct size following 2-hour reperfusion was likewise markedly reduced, from $53 \% \pm 3 \%$ in WT and $50 \% \pm 3 \%$ in tTA, to $20 \% \pm 1 \%$ in CA-RhoA mice (Figure 3C). No protection was seen in doubletransgenic mice maintained on Dox to suppress RhoA expression (Supplemental Figure 2). CA-RhoA hearts also showed resistance to mitochondrial damage, as indicated by diminished cytochrome $c$ accumulation in the cytosolic compartment (Figure 3, D and E).

CA-RhoA hearts show no alteration in activation of AKT, FAK, ERK, PKN,

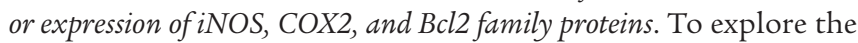
mechanism for RhoA-mediated protection, we looked for changes in activation or expression of potential RhoA downstream targets implicated in cardioprotection. CA-RhoA hearts at 8 weeks off Dox did not show increases in activated (phosphorylated) AKT, focal adhesion kinase (FAK), ERK, or PKN (also known as PKC-related kinase 1 or PRK1) compared with control hearts at baseline (Supplemental Figure 3). There were also no obvious changes in expression of $\mathrm{COX} 2$ and iNOS, antiapoptotic $\mathrm{Bcl} 2$ and $\mathrm{Bcl}-\mathrm{xl}$ proteins, or proapoptotic Bax and Bak proteins (Supplemental Figure 3). Rac1, a member of the Rho small GTPase family that is negatively regulated by RhoA (17), has been reported to protect against oxidative stress in noncardiomyocytes $(18,19)$. Activity and expression of Rac1 were unaltered, however, in CA-RhoA mouse hearts (Supplemental Figure 4). Thus, conventional and previously reported prosurvival signals were not affected in ways that would provide cardioprotection in the CA-RhoA mouse heart.

RhoA signaling to novel PKCs and PKD. PKC regulates many cardiac functions, including cardiomyocyte survival (20). To determine the activation status of conventional and novel PKC (nPKC) isozymes, we assessed the membrane and cytosolic localization of 3 representative $\mathrm{PKC}$ isozymes. The most abundant ventricular PKC isozyme, PKC $\alpha$ (a conventional PKC isozyme), did not show altered membrane localization in CA-RhoA versus control hearts. In contrast, membrane localization of $2 \mathrm{nPKC}$ isozymes, $\mathrm{PKC} \varepsilon$ and $\mathrm{PKC} \delta$, was increased (and cytosolic localization decreased) in hearts from CA-RhoA versus control mice (Figure 4A). PKD is an established target for nPKCs $(21,22)$, which catalyzes phosphorylation at sites in the acti- 
A
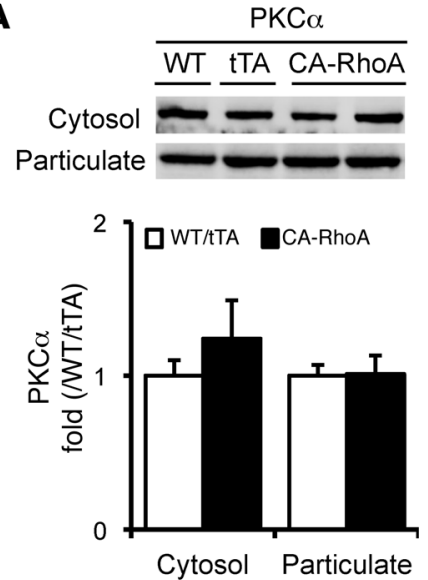
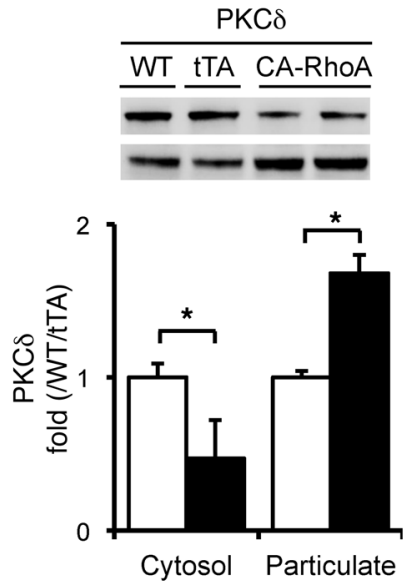
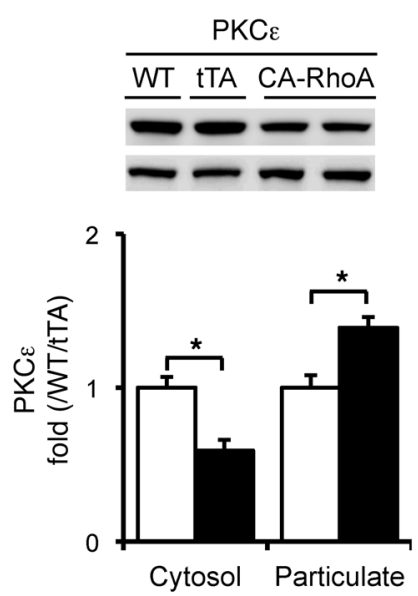

Figure 4

nPKC isozymes and PKD are activated in CA-RhoA hearts. (A) Representative blots and quantification of cytosolic and particulate $\mathrm{PKC} \alpha, \operatorname{PKC} \delta$, and $P K C \varepsilon$ fractionated from the LV of WT, tTA, and CA-RhoA hearts ( 8 weeks off Dox). ${ }^{*} P<0.01(n=3-6)$. (B) PKD phosphorylation (pPKD) and expression in LV lysates from WT, tTA, and CA-RhoA hearts ( 8 weeks off Dox). ${ }^{*} P<0.01(n=6)$. Data are shown as mean \pm SEM.

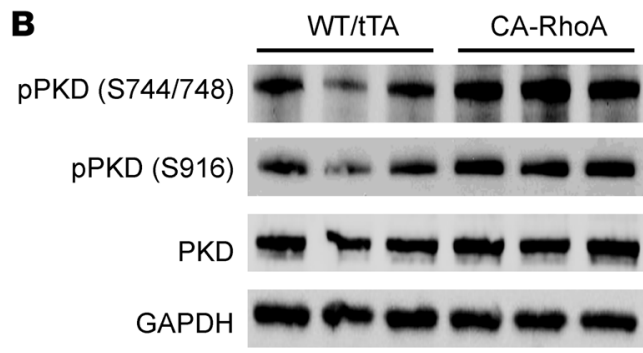

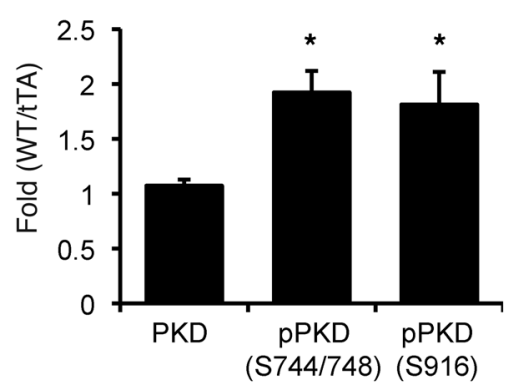

vation loop of PKD (Ser744/748), leading to its autophosphorylation at the extreme C terminus (Ser916) (21-24). Phosphorylation of both sites on PKD was markedly increased (by $90 \%$ and $81 \%$, respectively) in CA-RhoA compared with control mice (Figure 4B).

$R h o A$ and $P K D$ are activated during oxidative stress and induce protection against oxidative stress-induced cell death. To directly demonstrate that RhoA signaling can activate PKD in cardiomyocytes and to investigate the role of RhoA/PKD in oxidative stress-induced cell death, we carried out studies using neonatal rat ventricular myocytes (NRVMs). NRVMs infected with constitutively active (Q63L) RhoA showed robust increases in PKD phosphorylation (Figure 5A). Calpeptin, a pharmacological activator of endogenous RhoA, also increased PKD phosphorylation, and its effect was attenuated by pretreatment with $\mathrm{C} 3$ transferase (Figure $5 \mathrm{~B}$ ). which ribosylates and functionally inhibits RhoA (25). Both RhoA and PKD were found to be activated in NRVMs following oxidative stress induced by $\mathrm{H}_{2} \mathrm{O}_{2}$ (Figure $5 \mathrm{C}$ ). Importantly, we documented a protective role for these responses based on the observation that treatment with C3, with a selective PKD inhibitor CID755673 (26), or with PKD siRNA potentiated $\mathrm{H}_{2} \mathrm{O}_{2}$-induced cell death (Figure 5D).

$P K D$ inhibition reverses $R$ hoA-mediated protection against $I / R$ injury in the heart. We reported previously that $\mathrm{I} / \mathrm{R}$ activates RhoA in the isolated perfused mouse heart (5). Here we extended these findings by demonstrating that RhoA activation following $I / R$ is accompanied by phosphorylation of PKD (Figure 6). The PKD inhibitor CID755673 effectively decreased PKD phosphorylation when perfused into control or CA-RhoA hearts for 30 minutes (Supplemental Figure 5). Accordingly, we used CID755673 pretreatment to determine whether PKD activation serves a protective role during I/R. Infarct size and LDH release induced by I/R were both significantly increased in WT/tTA mouse hearts pretreated with CID755673 (Figure 7, A and B). Moreover, cardioprotection against I/R in the CA-RhoA hearts was completely reversed by PKD inhibition (Figure 7, A and B). These data support the conclusion that PKD activated during $\mathrm{I} / \mathrm{R}$ is cardioprotective and that $\mathrm{PKD}$ mediates the robust protective effect of RhoA in I/R injury.

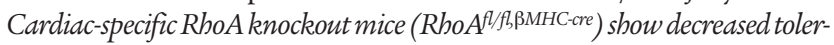
ance to I/R injury. To further support the conclusions reached through experiments using RhoA gain of function we obtained cardiac-specific RhoA gene-deleted mice (see Methods). Double-transgenic mice Rho Al/fl, $\mathrm{AMHC}$-cre showed more than $60 \%$ reduction in RhoA expression in the LV compared with their littermate $R b o A^{f / f l}$ controls (Figure 8A). The remaining RhoA protein likely reflects RhoA expression in other cardiac cell types (e.g., fibroblasts, vascular endothelial cells, and inflammatory cells) as well as incomplete Cre-mediated excision in cardiomyocytes. PKD expression and phosphorylation in $R h o A^{f l / f l, \beta M H C-c r e ~}$ mouse hearts were unchanged at baseline relative to control hearts, but PKD autophosphorylation following I/R was significantly attenuated in the hearts in which RhoA was genetically deleted (Figure 8B). Furthermore, RhoA $A^{f l f l, \beta M H C-c r e}$ mouse hearts showed significantly increased LDH release and infarct size following I/R compared with their WT, ${ }^{\beta} \mathrm{MHC}$-cre, and $R h o A^{\ell / / f l}$ controls (Figure 8, $\mathrm{C}$ and $\mathrm{D})$. These data indicate that RhoA disruption in cardiomyocytes increases I/R injury and thereby confirm a cardioprotective role for RhoA and PKD signaling in myocytes during I/R.

\section{Discussion}

The small GTPase RhoA plays an important role in transducing signals from the extracellular matrix and from a subset of $G$ protein-coupled receptors, but its role in regulating cardiac physiology and pathophysiology has remained elusive. Early studies suggested that RhoA and its downstream effector ROCK contribute to development of cardiac hypertrophy $(9,10,27-30)$. We have observed, however, that while chronic expression of activated RhoA begin- 
A

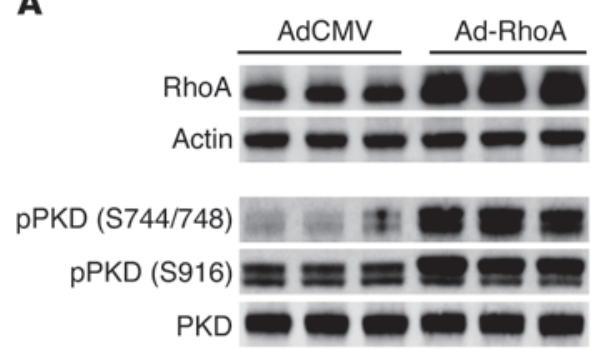

C

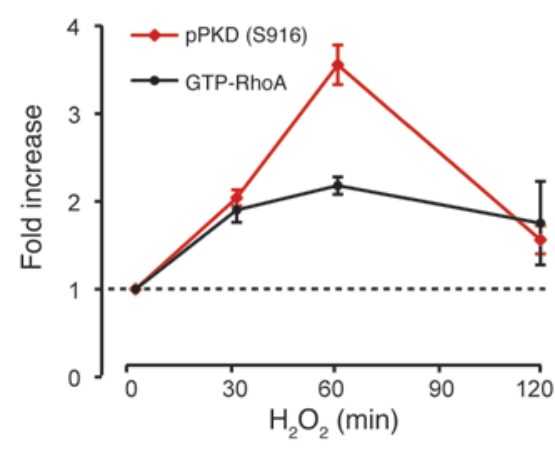

B

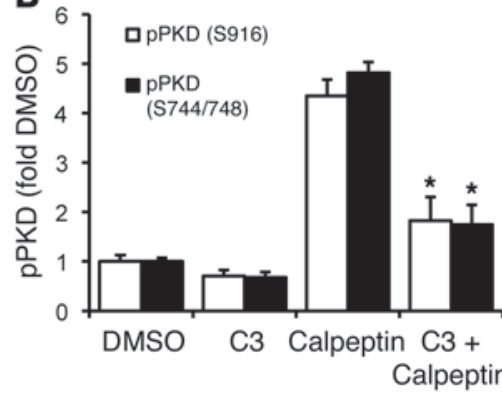

D
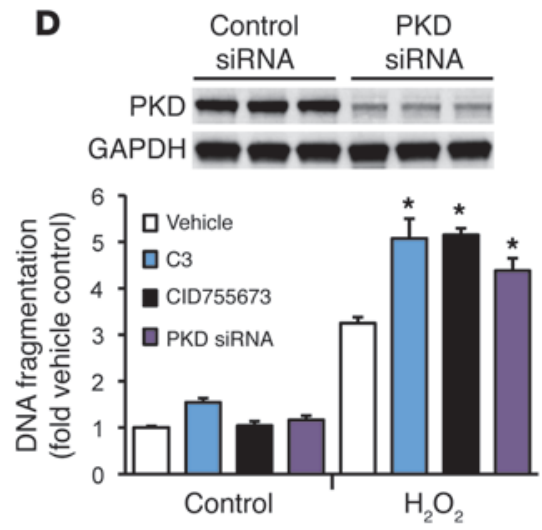

Figure 5

RhoA induces PKD activation in NRVMs. (A) Adenoviral expression of RhoA induces PKD phosphorylation. (B) A Rho activator calpeptin (1 unit/ml, 30 minutes) induces PKD phosphorylation, which is inhibited by RhoA inactivation with C3 $(2 \mu \mathrm{g} / \mathrm{ml}$, 18 hours). ${ }^{*} P<0.05$ versus calpeptin $(n=3-4)$. (C) Time course activation of RhoA and PKD in response to $\mathrm{H}_{2} \mathrm{O}_{2}$ $(150 \mu \mathrm{M})$ treatment $(n=3-5)$. (D) Top panel: PKD knockdown by siRNA $(90 \%$ knockdown). Bottom panel: treatment with C3 $(1 \mu \mathrm{g} / \mathrm{ml})$, CID755673 (50 $\mu \mathrm{M})$, or PKD siRNA potentiates cell death induced by $\mathrm{H}_{2} \mathrm{O}_{2}$ ( $150 \mu \mathrm{M}, 20$ hours). ${ }^{*} P<0.05$ versus vehicle $\mathrm{H}_{2} \mathrm{O}_{2}(n=4-5)$. Data are shown as mean \pm SEM. ning in early development can induce hypertrophy (Supplemental Figure 1) and that a lethal dilated cardiomyopathy and heart failure develop with chronic high level expression of RhoA (7), this does not occur in mice expressing low levels of RhoA in the adult heart. The CA-RhoA lines generated and examined in the current study show conditional (upon Dox removal) and modest (2- to 5-fold) increases in active GTP-RhoA, increases comparable to those induced in the mouse heart by treatment with agonists such as sphingosine-1-phosphate (13) or by I/R (Figure 6). Oxidative stress or inflammatory mediators released during ischemia and reperfusion would be expected to activate RhoA.Thus, our experimental model mimics and allows us to question the role that RhoA serves when the heart is initially exposed to I/R. Importantly, because RhoA is specifically expressed in cardiomyocytes in CA-RhoA mice and specifically deleted from cardiomyocytes in the RhoA-knockout mice, we can resolve the effects of RhoA in cardiomyocytes from its potential effects in other cell types in the heart. Our data clearly demonstrate that a modest level of RhoA activation in adult cardiomyocytes is not pathological but, remarkably, defines a previously unrecognized pathway for cardioprotection.

When CA-RhoA mouse hearts were challenged by I/R, we observed profound tolerance to both ex vivo and in vivo I/R injury. Protection was manifest as $60 \%$ to $70 \%$ reductions in infarct size, decreased $\mathrm{LDH}$ release, and markedly improved postischemic contractile performance. Parallel loss-of-function experiments using cardiac-specific RhoA-knockout mice demonstrated markedly increased infarct size and LDH release when cardiomyocytes did not express RhoA. These data provide substantial support for the conclusion that endogenous RhoA is activated in cardiomyocytes during $\mathrm{I} / \mathrm{R}$, where it functions to protect myocytes against I/R injury.

Figure 6
Further studies examined the mechanism by which RhoA mediates cardioprotection. In the CA-RhoA heart, the autophosphorylation of PKD was markedly increased, indicative of its activation. RhoA expression or RhoA activation in NRVMs also increased PKD phosphorylation. These data extend previously published work showing that PKD is activated when RhoA is heterologously expressed in COS-7 and HeLa cells $(31,32)$. There is also increased membrane distribution of PKCE and PKC $\delta$ in CA-RhoA hearts. These nPKC isozymes are known to phosphorylate and activate $\operatorname{PKD}(22,23)$, and indeed we observed increased phosphorylation of established PKC sites on PKD in the CA-RhoA mouse heart.

There is, to our knowledge, no previous evidence that PKD mediates cardioprotection. Accordingly, we used inhibitors of PKD to test its role in the cardioprotection observed in the CA-RhoA
A

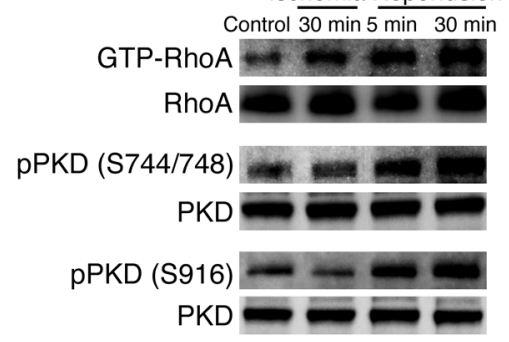

B

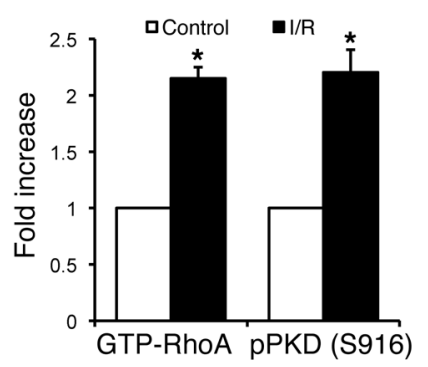

RhoA and PKD are activated in response to ex vivo $I / R$ in the heart. Effects of $I / R$ on RhoA activation and PKD phosphorylation. (A) Representative blots showing time course of RhoA activation and PKD phosphorylation in response to I/R in the isolated perfused WT mouse hearts. (B) Quantification of change in RhoA activation and $\mathrm{PKD}$ phosphorylation by 30 -minute ischemia followed by 30 -minute reperfusion. ${ }^{\star} P<0.05$ versus preischemic control $(n=3$ to 4$)$. Data are shown as mean \pm SEM. 


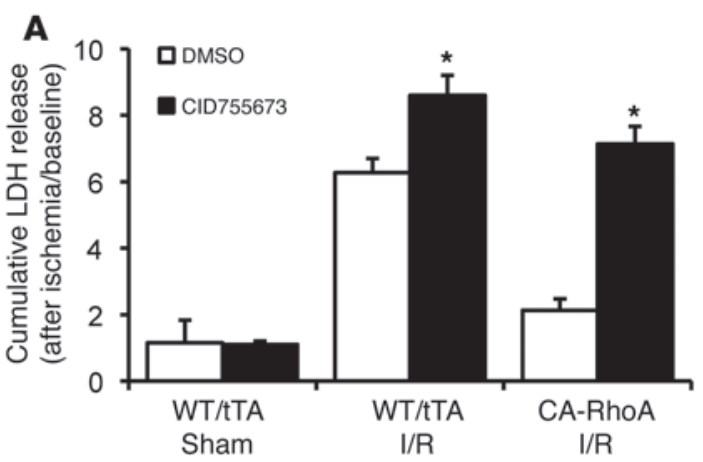

B

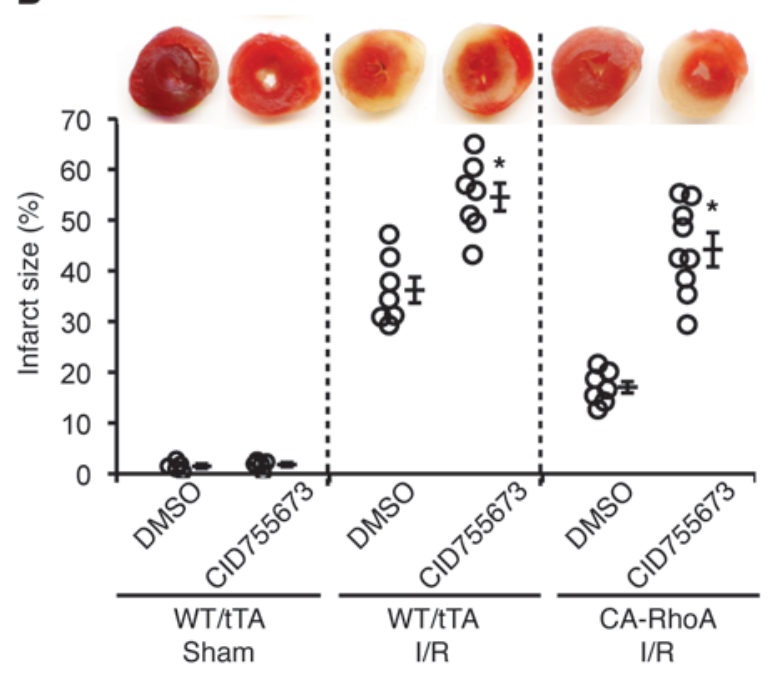

Figure 7

PKD inhibition enhances I/R injury and reverses cardioprotection in the CA-RhoA heart. WT, tTA, and CA-RhoA hearts were perfused with PKD inhibitor CID755673 (50 $\mu \mathrm{M})$ or DMSO for 30 minutes before I/R. (A) LDH release to the coronary effluent following 45-minute reperfusion. ${ }^{*} P<0.005$ versus DMSO $(n=5-9)$. (B) Representative TTC-stained ventricular sections (top); quantitative analysis of infarct size (bottom). ${ }^{*} P<0.005$ versus DMSO $(n=5-9)$. Sham, WT/tTA mouse hearts subjected to perfusion without ischemia with DMSO or CID755673. Data are shown as mean \pm SEM.

mouse heart. Either of 2 classes of PKD inhibitor significantly reversed cardioprotection against I/R injury (Figure 7 and Supplemental Figure 7). We further demonstrate that both RhoA and $\mathrm{PKD}$ are activated in response to oxidative stress induced by $\mathrm{I} / \mathrm{R}$ in the WT mouse heart and that I/R injury in the WT mouse heart is exacerbated by inhibition of PKD. Finally, we provide evidence that the PKD activation that occurs with $\mathrm{I} / \mathrm{R}$ is prevented when RhoA is genetically deleted from cardiomyocytes, and this is accompanied by increased infarct size and LDH release. Studies using NRVMs additionally show that $\mathrm{H}_{2} \mathrm{O}_{2}$ activates RhoA and PKD and that acute loss of function of RhoA or PKD by siRNA or pharmacological inhibitors increases $\mathrm{H}_{2} \mathrm{O}_{2}$-induced DNA fragmentation. Taken together, these data implicate RhoA and PKD in a previously unexplored protective signaling pathway to counteract the deleterious effects of oxidative stress on cardiomyocytes.

It is of note that RhoA and PKD, identified here as what we believe to be novel players in cardioprotection, could be involved in the pathways utilized by more established cardioprotective mediators. For example, nPKCs, particularly PKCe, mediate cardioprotection $(33,34)$. As a downstream target for phosphorylation by the nPKC isozymes $(22,23)$, PKD could contribute to their protective effects. The effects of the Rho-activated kinase, ROCK, in cardiac pathophysiology also bear comment, since several laboratories have reported salutary effects of inhibiting or deleting ROCK, suggesting that Rho activation in the heart is deleterious $(5,30,35)$. In this regard, a key element of the data presented here is that our models have allowed us to focus on what RhoA does in the cardiomyocyte, distinct from the potential ROCK-mediated functions of RhoA in fibroblasts $(30,36)$, arterial smooth muscle cells (37-39), or endothelial cells $(40,41)$, where proliferation or membrane permeability changes would be maladaptive. The unexpected cardioprotective role of RhoA in cardiomyocytes, taken with evidence that it is activated at early times following reperfusion, suggests that RhoA is not always a villain and that one may want to facilitate rather than block its activation at early times during reperfusion of the ischemic heart.

In conclusion, our studies demonstrate that modest levels of RhoA activation in adult mouse cardiomyocytes provide striking protection against I/R injury and that genetic deletion of RhoA in cardiomyocytes renders the heart more susceptible to I/R damage. Our findings reveal a previously undescribed signaling pathway for RhoA in the heart and suggest that PKD mediates cardioprotection. These new insights into the effects of RhoA in I/R injury suggest that interventions that activate RhoA could play an important physiological or therapeutic role in promoting cardiomyocyte survival.

\section{Methods}

An expanded methods section is available in the Supplemental Materials and Methods. All procedures were performed in accordance with the NIH Guide for the Care and Use of Laboratory Animals and approved by the Institutional Animal Care and Use Committee of UCSD.

Generation of cardiac-specific inducible RhoA transgenic (CA-RhoA) mice. Transgenic mice were generated in the Dorn laboratory using the tet-off system developed by the Robbins laboratory (16). For the responder mouse line, HA-tagged human constitutively activated RhoA (a gift from G. Bokoch, The Scripps Research Institute, La Jolla, California, USA) was cloned into a modified $\alpha \mathrm{MHC}$ promoter construct that allows tetracycline/Dox responsive expression (Dox-off) in the heart when crossed with the driver transgenic mouse line carrying the $\alpha \mathrm{MHC}$-driven $\mathrm{tTA}$. Both the responder (modified $\alpha$-MHC-RhoA) line and the driver line ( $\alpha$ MHC-tTA) are in the FVB/N background. Dox was administered in the food until 3 weeks after birth to inhibit transgenic RhoA expression and to eliminate developmental effects of RhoA. Dox was removed after 3 weeks of age to induce cardiac-specific expression of transgenic RhoA.

RhoA activation assay. RhoA activation was determined by affinity pulldown assay using a glutathione $S$-transferase (GST) fusion protein of the RhoA binding domain of the RhoA effector rhotekin, as described previously by our group (42). Freshly isolated LVs were rinsed with ice-cold Tris-buffered saline, lysed in buffer containing $50 \mathrm{mM}$ Tris, $\mathrm{pH} 7.4,0.1 \%$ Triton X-100, $150 \mathrm{mM}, \mathrm{NaCl}, 5 \mathrm{mM} \mathrm{MgCl}_{2}$, and $10 \%$ glycerol, supplemented with protease and phosphatase inhibitors, clarified by brief centrifugation, and then incubated with the sepharose-bound GST-rhotekin-RhoA binding domain for 45 minutes at $4{ }^{\circ} \mathrm{C}$. The beads and precipitated proteins were washed, boiled, and separated by SDS-PAGE. The precipitated GTP-bound RhoA was normalized to total RhoA present in the whole cell (or tissue) lysate.

Histological analysis. Tissues were fixed in $4 \%$ paraformaldehyde/PBS, dehydrated in $70 \%$ ethanol, embedded in paraffin wax, and sectioned. Paraffin sections were stained with H\&E or Tri. To determine the size of cardiomyocytes, transverse paraffin sections were labeled with tetramethyl 
A

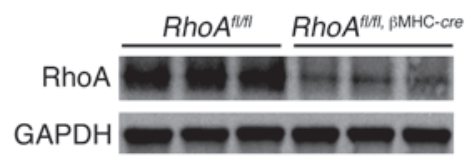

B
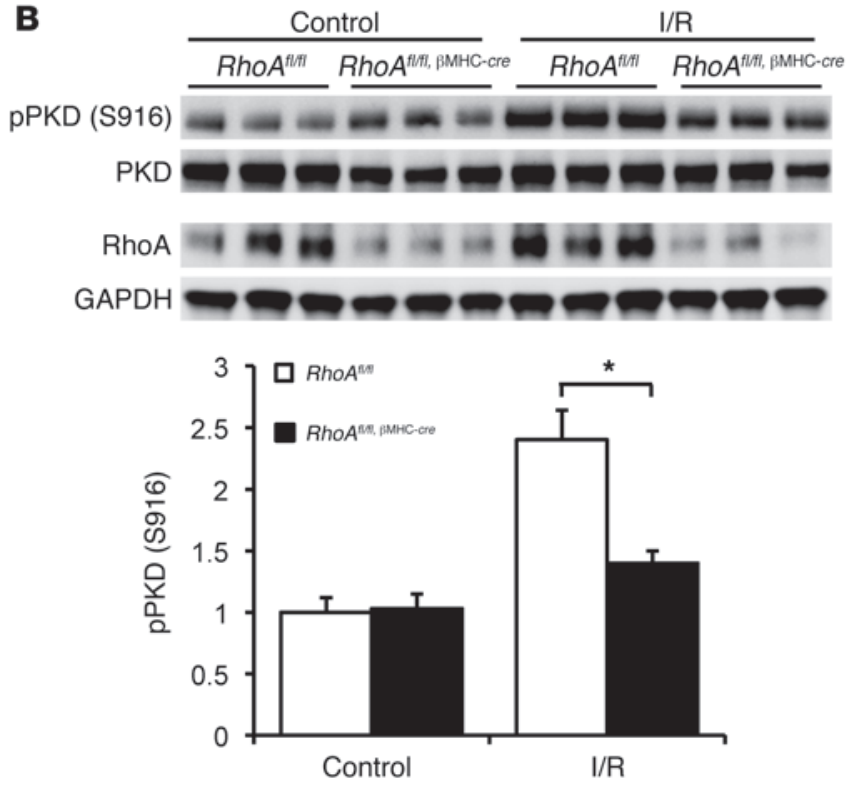

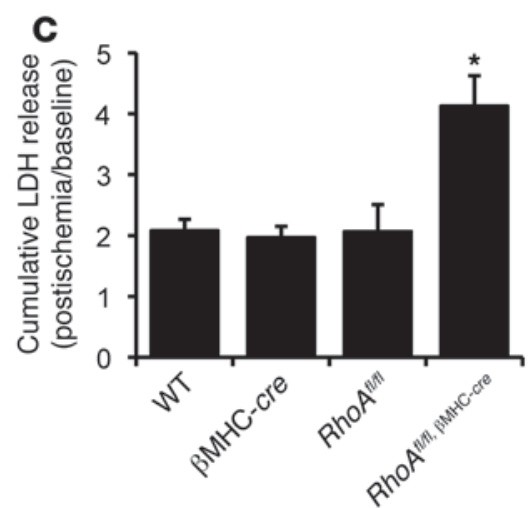

D
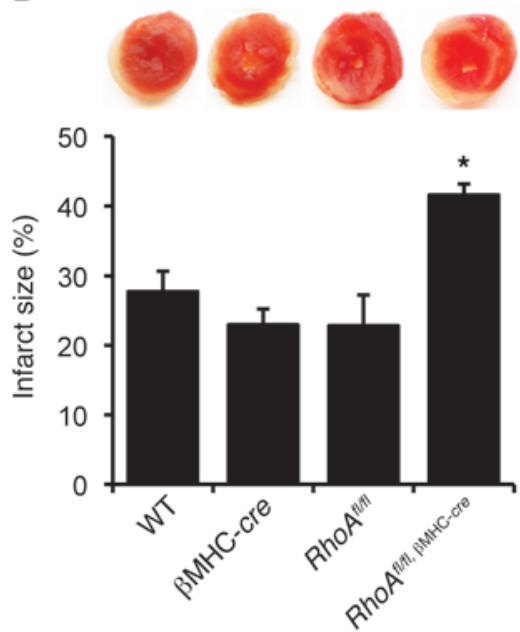

Figure 8

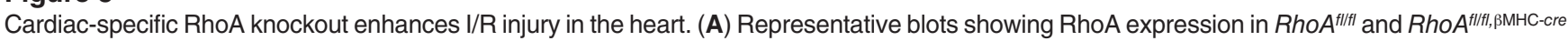
hearts. (B) Representative blots and quantification showing PKD autophosphorylation in $R h \circ A^{f / / f l}$ and $R h o A^{f / f t}, \beta M H C$-cre hearts following l/R. ${ }^{*} P<0.05$

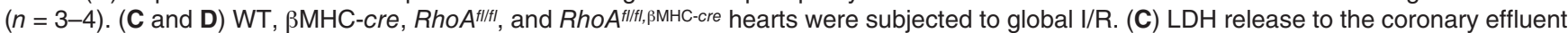
following 45-minute reperfusion. ${ }^{*} P<0.05$ versus controls $(n=4-5)$. (D) Representative TTC-stained ventricular sections (top); quantitative analysis of infarct size (bottom). ${ }^{*} P<0.05(n=4-7)$. Data are shown as mean \pm SEM.

rhodamine isothiocyanate-labeled (TRITC-labeled) wheat germ agglutinin (WGA) (Sigma-Aldrich), nuclei were counterstained with DAPI (Vector Laboratories), and the cross-sectional area of myocytes in the LV wall was determined using Image Pro Plus software as described previously (7).

In vivo $I / R$. Coronary occlusion/reperfusion was performed as described in detail in previous studies (43). Age-matched male mice were anesthetized by intraperitoneal injection of pentobarbital sodium $(60 \mathrm{mg} / \mathrm{kg})$, intubated, and ventilated with $65 \%$ oxygen during the surgery. LAD was occluded at a position $2 \mathrm{~mm}$ from the tip of the left auricle. Myocardial ischemia was verified by blanching of the $\mathrm{LV}$ and by changes in electrocardiogram. Following 1-hour LAD occlusion and 24-hour reperfusion, the LAD was reoccluded and $5 \%$ Evans blue dye $(0.2 \mathrm{ml})$ was injected into the $\mathrm{LV}$ cavity to define the nonischemic zone. The ventricles were frozen and cut transversely into 5 slices of equal thickness followed by incubation in $1 \%$ triphenyltetrazolium chloride (TTC)/PBS at $37^{\circ} \mathrm{C}$ for 8 minutes and then were fixed in $10 \%$ formalin-PBS for 24 hours. Fixed slices were then scanned, and Image software (NIH) was used to measure and calculate the $\mathrm{AAR}$, infarct tissue, and the total area.

Isolated perfused (ex vivo) $I / R$. Hearts from age-matched male mice were removed quickly and perfused in a retrograde manner with modified KrebsHenseleit buffer (118 mM NaCl, $4.7 \mathrm{mM} \mathrm{KCl}, 1.2 \mathrm{mM} \mathrm{KH}_{2} \mathrm{PO}_{4}, 25 \mathrm{mM}$
$\mathrm{NaHCO}_{3}, 0.5 \mathrm{mM}$ EDTA, $1.2 \mathrm{mM} \mathrm{MgSO} 4,11 \mathrm{mM}$ glucose, $1.5 \mathrm{mM} \mathrm{Na}-$ Pyruvate, and $2 \mathrm{mM} \mathrm{CaCl}_{2}$ ) in a Langendorff apparatus at constant pressure $(80 \mathrm{mmHg})$. To record cardiac contractile function, a water-filled balloon made of plastic film connected to a pressure transducer was inserted into the LV. After stabilization, hearts were subjected to 30 minutes of global ischemia, followed by 60 minutes of reperfusion. The LVDP (peak systolic pressure minus LV end diastolic pressure [LVEDP]), $+\mathrm{dP} / \mathrm{dt}$, and $-\mathrm{dP} / \mathrm{dt}$ were monitored and recorded. To measure infarct size, hearts were subjected to 45-minute global ischemia and 2-hour reperfusion; the ventricles were then frozen and cut transversely into 5 slices of equal thickness. For the cardiac-specific RhoA knockout $\left(R h o A^{f / / f l \beta M H C-c r e}\right)$ mice and their control mice, the ischemia time was adjusted to 25 minutes. The slices were then incubated in $1 \%$ TTC/PBS and fixed in $10 \%$ formalin-PBSe for 24 hours. Fixed slices were then scanned, and ImageJ was used to measure and calculate the size of the infarct area and the total area.

LDH activity assay. To measure LDH release during reperfusion, hearts were subjected to 45 -minute global ischemia and 2-hour reperfusion in the Langendorff mode. Coronary outflow was collected during the last $10 \mathrm{~min}$ utes of baseline perfusion and the first 45 -minute reperfusion following ischemia; the volume was measured and the cumulated LDH release was determined using an LDH activity assay kit (MBL International Corp.). 
Generation of cardiac-specific RhoA-knockout (RhoA Al/f,BMHC-cre) mice. The cardiac-specific RhoA knockout mice were generated in the Molkentin laboratory using the RhoA loxP-targeted ( $f l$ ) mice generated in Yi Zheng's laboratory, in which exon 3 was flanked by loxP sites to allow tissue-specific deletion, as described in Supplemental Figure 6. Mice expressing Cre recombinase under control of the endogenous ${ }^{\beta} \mathrm{MHC}$ promoter were described previously (44). Mice harboring the $R b o A^{f / f l}$ alleles and ${ }^{\beta}$ MHC-cre transgenic mice, independently generated from the same back-cross (C57BL/6 - SV129), were crossed to generate $R h o A^{f / f f l, \beta M H C-c r e}$ mice in the Molkentin laboratory.

Statistics. All results are reported as mean \pm SEM. Comparison of 2 groups was accomplished using an unpaired Student's $t$ test. Data from experiments with more than 2 groups were compared by 1-way ANOVA followed by the Tukey post hoc test for comparison between groups. $P<0.05$ was considered to be statistically significant.

1. Sander EE, Collard JG. Rho-like GTPases: their role in epithelial cell-cell adhesion and invasion. Eur J Cancer. 1999;35(14):1905-1911.

2. Ai S, et al. Rho-Rho kinase is involved in smooth muscle cell migration through myosin light chain phosphorylation-dependent and independent pathways. Atherosclerosis. 2001;155(2):321-327.

3. Zhang S, et al. RhoA regulates $\mathrm{G} 1-\mathrm{S}$ progression of gastric cancer cells by modulation of multiple INK4 family tumor suppressors. Mol Cancer Res. 2009;7(4):570-580.

4. Ueyama T, et al. Activated RhoA stimulates c-fos gene expression in myocardial cells. Circ Res. 1997;81(5):672-678.

5. Miyamoto S, Del Re DP, Xiang SY, Zhao X, Florholmen G, Brown JH. Revisited and revised: is RhoA always a villain in cardiac pathophysiology? J Cardiovasc Transl Res. 2010;3(4):330-343.

6. Del Re DP, Miyamoto S, Brown JH. RhoA / Rho kinase upregulate Bax to activate a mitochondrial death pathway and induce cardiomyocyte apoptosis. J Biol Chem. 2007;282(11):8069-8078.

7. Sah VP, et al. Cardiac-specific overexpression of RhoA results in sinus and atrioventricular nodal dysfunction and contractile failure. J Clin Invest. 1999;103(12):1627-1634.

8. Kontaridis MI, et al. Deletion of Ptpn11 (Shp2) in cardiomyocytes causes dilated cardiomyopathy via effects on the extracellular signal-regulated kinase/mitogen-activated protein kinase and RhoA signaling pathways. Circulation. 2008; 117(11):1423-1435.

9. Phrommintikul A, et al. Effects of a Rho kinase inhibitor on pressure overload induced cardiac hypertrophy and associated diastolic dysfunction. Am J Physiol Heart Circ Physiol. 2008;294(4):H1804-H1814.

10. Higashi $M$, et al. Long-term inhibition of Rhokinase suppresses angiotensin II-induced cardiovascular hypertrophy in rats in vivo: effect on endothelial NAD(P)H oxidase system. Circ Res. 2003; 93(8):767-775

11. Shi J, Zhang L, Wei L. Rho-kinase in development and heart failure: insights from genetic models. Pediatr Cardiol. 2011;32(3):297-304.

12. Dong M, Yan BP, Liao JK, Lam YY, Yip GW, Yu CM. Rho-kinase inhibition: a novel therapeutic target for the treatment of cardiovascular diseases. Drug Discov Today. 2010;15(15-16):622-629.

13. Del Re DP, Miyamoto S, Brown JH. Focal adhesion kinase as a RhoA-activable signaling scaffold mediating Akt activation and cardiomyocyte protection. J Biol Chem. 2008;283(51):35622-35629.

14. Krijnen PA, et al. Inhibition of Rho-ROCK signaling induces apoptotic and non-apoptotic PS exposure in cardiomyocytes via inhibition of flippase. J Mol Cell Cardiol. 2010;49(5):781-790.

15. Mozzicato S, Joshi BV, Jacobson KA, Liang BT. Role of direct RhoA-phospholipase D1 interaction in mediating adenosine-induced protection from

\section{Acknowledgments}

We thank Melissa Ridout for support with animal breeding. This work was supported by NIH grant RO1 HL28143 and Cardiovascular Physiology and Pharmacology Training grant 5T32HL007444. D. Vanhoutte is supported by a postdoctoral fellowship from the Research Foundation - Flanders (FWOVlaanderen).

Received for publication April 26, 2011, and accepted May 18, 2011.

Address correspondence to: Joan Heller Brown, Department of Pharmacology, University of California San Diego, 9500 Gilman Dr., La Jolla, California 92093-0636, USA. Phone: 858.822.5858; Fax: 858.822.0041; E-mail: jhbrown@ucsd.edu.

cardiac ischemia. FASEB J. 2004;18(2):406-408

16. Sanbe A, Gulick J, Hanks MC, Liang Q, Osinska $\mathrm{H}$, Robbins J. Reengineering inducible cardiac-specific transgenesis with an attenuated myosin heavy chain promoter. Circ Res. 2003;92(6):609-616.

17. Sugimoto N, Takuwa N, Yoshioka K, Takuwa Y Rho-dependent, Rho kinase-independent inhibitory regulation of Rac and cell migration by LPA1 receptor in Gi-inactivated CHO cells. Exp Cell Res. 2006;312(10):1899-1908.

18. Murga C, Zohar M, Teramoto H, Gutkind JS. Rac1 and RhoG promote cell survival by the activation of PI3K and Akt, independently of their ability to stimulate JNK and NF-kappaB. Oncogene. 2002;21(2):207-216.

19. Zhang B, Zhang Y, Shacter E. Rac1 inhibits apoptosis in human lymphoma cells by stimulating Bad phosphorylation on Ser-75. Mol Cell Biol. 2004; 24(14):6205-6214.

20. Palaniyandi SS, Sun L, Ferreira JC, Mochly-Rosen D. Protein kinase $\mathrm{C}$ in heart failure: a therapeutic target? Cardiovasc Res. 2009;82(2):229-239.

21. Rey O, Reeve JR Jr, Zhukova E, Sinnett-Smith J, Rozengurt E. G protein-coupled receptor-mediated phosphorylation of the activation loop of protein kinase D: dependence on plasma membrane translocation and protein kinase Cepsilon. J Biol Chem. 2004;279(33):34361-34372.

22. Tan M, Xu X, Ohba M, Ogawa W, Cui MZ. Thrombin rapidly induces protein kinase $\mathrm{D}$ phosphorylation, and protein kinase $\mathrm{C}$ delta mediates the activation. J Biol Chem. 2003;278(5):2824-2828.

23. Brandlin I, Eiseler T, Salowsky R, Johannes FJ. Protein kinase $\mathrm{C}(\mathrm{mu})$ regulation of the JNK pathway is triggered via phosphoinositide-dependent kinase 1 and protein kinase C(epsilon).J Biol Chem. 2002;277(47):45451-45457.

24. Matthews SA, Rozengurt E, Cantrell D. Characterization of serine 916 as an in vivo autophosphorylation site for protein kinase D/Protein kinase Cmu. J Biol Chem. 1999;274(37):26543-26549.

25. Aktories K, Rosener S, Blaschke U, Chhatwal GS. Botulinum ADP-ribosyltransferase C3: purification of the enzyme and characterization of the ADP-ribosylation reaction in platelet membranes. Eur J Biochem. 1988;172(2):445-450.

26. Sharlow ER, et al. Potent and selective disruption of protein kinase D functionality by a benzoxoloazepinolone. J Biol Chem. 2008;283(48):33516-33526.

27. Brown JH, Del Re DP, Sussman MA. The Rac and Rho hall of fame: a decade of hypertrophic signaling hits. Circ Res. 2006;98(6):730-742.

28. Sah VP, Hoshijima M, Chien KR, Brown JH. Rho is required for Galphaq and alpha1-adrenergic receptor signaling in cardiomyocytes. Dissociation of Ras and Rho pathways. J Biol Chem. 1996; 271(49):31185-31190.

29. Morissette MR, Sah VP, Glembotski CC, Brown JH. The Rho effector, PKN, regulates ANF gene transcription in cardiomyocytes through a serum response element. Am J Physiol Heart Circ Physiol. 2000;278(6):H1769-H1774.

30. Zhang YM, et al. Targeted deletion of ROCK1 protects the heart against pressure overload by inhibiting reactive fibrosis. FASEB J. 2006;20(7):916-925.

31. Yuan J, Slice LW, Rozengurt E. Activation of protein kinase D by signaling through Rho and the alpha subunit of the heterotrimeric G protein G13.J Biol Chem. 2001;276(42):38619-38627.

32. Cowell CF, Yan IK, Eiseler T, Leightner AC, Doppler $\mathrm{H}$, Storz P. Loss of cell-cell contacts induces NFkappaB via RhoA-mediated activation of protein kinase D1. J Cell Biochem. 2009;106(4):714-728.

33. Liu GS, Cohen MV, Mochly-Rosen D, Downey JM. Protein kinase C-epsilon is responsible for the protection of preconditioning in rabbit cardiomyocytes. J Mol Cell Cardiol. 1999;31(10):1937-1948.

34. Baines CP, et al. Protein kinase Cepsilon interacts with and inhibits the permeability transition pore in cardiac mitochondria. Circ Res. 2003;92(8):873-880.

35. Hamid SA, Bower HS, Baxter GF. Rho kinase activation plays a major role as a mediator of irreversible injury in reperfused myocardium. Am J Physiol Heart Circ Physiol. 2007;292(6):H2598-H2606.

36. Haudek SB, et al. Rho kinase- 1 mediates cardiac fibrosis by regulating fibroblast precursor cell differentiation. Cardiovasc Res. 2009;83(3):511-518.

37. Guilluy C, et al. The Rho exchange factor Arhgef1 mediates the effects of angiotensin II on vascular tone and blood pressure. Nat Med. 2010;16(2):183-190.

38. Masumoto A, Mohri M, Shimokawa H, Urakami L, Usui M, Takeshita A. Suppression of coronary artery spasm by the Rho-kinase inhibitor fasudil in patients with vasospastic angina. Circulation. 2002;105(13):1545-1547.

39. Zhou Q, Gensch C, Liao JK. Rho-associated coiledcoil-forming kinases (ROCKs): potential targets for the treatment of atherosclerosis and vascular disease. Trends Pharmacol Sci. 2011;32(3):167-173.

40. Nohria A, et al. Rho kinase inhibition improves endothelial function in human subjects with coronary artery disease. Circ Res. 2006;99(12):1426-1432.

41. Noma K, et al. ROCK1 mediates leukocyte recruitment and neointima formation following vascular injury. J Clin Invest. 2008;118(5):1632-1644.

42. Sagi SA, Seasholtz TM, Kobiashvilla M, Wilson BA, Toksoz D, Brown JH. Physical and functional interactions of $\mathrm{G}_{\alpha \mathrm{q}}$ with Rho and its exchange factors. J Biol Chem. 2001;276(18):15445-15452.

43. Means CK, et al. Sphingosine 1-phosphate S1P2 and S1P3 receptor-mediated Akt activation protects against in vivo myocardial ischemia-reperfusion injury. Am J Physiol Heart Circ Physiol. 2007; 292(6):H2944-H2951.

44. Parsons SA, et al. Genetic loss of calcineurin blocks mechanical overload-induced skeletal muscle fiber type switching but not hypertrophy.JBiol Chem. 2004; 279(25):26192-26200 